ISSN 0103-9954

\title{
ATRIBUTOS ECOLÓGICOS DA BIOINVASORA Nicotiana glauca GRAHAM (SOLANACEAE) E AVALIAÇÃO DA SUSCEPTIBILIDADE DE SUA OCORRÊNCIA NO BRASIL
}

\author{
ECOLOGICAL ATTRIBUTES OF ALIEN Nicotiana glauca GRAHAM (SOLANACEAE) AND \\ ASSESSMENT OF THE SUSCEPTIBILITY OF THE SPECIES OCCURRING IN BRAZIL
}

\author{
Juliano Ricardo Fabricante ${ }^{1}$ Raphaela Aguiar de Castro ${ }^{2}$ Kelianne Carolina Targino de Araújo ${ }^{3}$ \\ José Alves de Siqueira-Filho ${ }^{4}$
}

\begin{abstract}
RESUMO
Nativa da Bolívia eArgentina, Nicotiana glauca Graham, atualmenteé encontrada crescendo espontaneamente em zonas áridas e semiáridas de diferentes partes do globo, sendo que, em alguns países, é considerada uma importante exótica invasora. No Brasil, a espécie ainda não havia sido reportada como invasora, mas nas áreas do Projeto de integração do Rio São Francisco (PISF), Nicotiana glauca pode ser observada formando aglomerados de indivíduos com altas densidades (mais de 37.000 indivíduos ha). Com isso, o objetivo do presente estudo foi inferir sobre a situação citada por meio de aspectos da estrutura (densidade, biometria e diversidade), reprodução (produção de frutos, propágulos e germinabilidade), presença de substâncias alelopáticas (extratos com concentrações de $0,5,10,15$ e 20\%) e da susceptibilidade de ocorrência da espécie no Brasil. A densidade estimada para a espécie foi de 37.280土2.442,3 indivíduos ha. A planta pode produzir mais de 1.300 .000 sementes por indivíduo. A partir da primeira concentração dos extratos (5\%), a germinação das sementes, tamanho e biomassa de plântulas de Lactuca sativa foram afetados significativamente. A espécie apresentou alta probabilidade de ocorrência nas regiões de clima semiárido e subúmido do Brasil. Os resultados deste estudo demonstram a necessidade do controle imediato da espécie nas áreas do PISF e, subsidiam a formulação de meios para evitar novos casos de invasão biológica por Nicotiana glauca em outras partes do Brasil.
\end{abstract}

Palavras-chave: bioinvasão; Caatinga; charuto-do-rei; Savana Estépica.

\section{ABSTRACT}

Native to Bolivia and Argentina, Nicotiana glauca Graham, is now found growing wild in arid and semiarid regions of different parts of the globe, where in some countries is an important exotic invasive. In Brazil the species had not been reported as invasive, but in the areas of design integration of the Rio São Francisco (PISF), Nicotiana glauca forming aggregates can be observed in individuals with high densities. Thus, this study sought to answer some questions in order to infer the situation observed in the Semiarid Northeast. We evaluated the structure, reproduction, existence of allelochemicals and ecological niche modeling of species. The estimated density for the species was $37,280 \pm 2442.3$ individuals $\mathrm{ha}^{-1}$. The plant can produce more than 1.3 million seeds per individual. From the initial concentration of the extract (5\%), seed germination, seedling size and biomass were significantly affected. The species had a high probability of occurring in regions of sub-humid and semi-arid climate of Brazil. The results of this study demonstrate the need for immediate control of the species in the PISF and subsidize the rationalization of resources to prevent new

1 Biólogo, Dr., Professor da Universidade Federal de Sergipe, Campus Universitário Prof. Alberto Carvalho, Departamento de Biociências, Av. Vereador Olímpio Grande, S/N - Bloco D, Centro, CEP: 49.500-000, Itabaiana (SE), Brasil. julianofabricante@hotmail.com

2 Graduanda em Engenharia Agronômica, Universidade Federal do Vale do São Francisco, BR 407, Km 12, Lote 543, s/n, Projeto Nilo Coelho - C1, CEP 56300-000, Petrolina (PE), Brasil. rapha.aguiarcastro@hotmail.com

3 Graduanda em Ciências Biológicas, Universidade Federal do Vale do São Francisco, BR 407, Km 12, Lote 543, s/n, Projeto Nilo Coelho - C1, CEP 56300-000, Petrolina (PE), Brasil. kelikarolina@hotmail.com

4 Biólogo, Dr., Professor da Universidade Federal do Vale do São Francisco, BR 407, Km 12, Lote 543, s/n, Projeto Nilo Coelho - C1, CEP 56300-000, Petrolina (PE), Brasil. jose.siqueira@univasf.edu.br

Recebido para publicação em 4/09/2013 e aceito em 18/12/2013

Ci. Fl., v. 25, n. 4, out.-dez., 2015 
cases of biological invasion by Nicotiana glauca in other parts of Brazil.

Keywords: bioinvasion; cigar-the-king; dry forest; Savannah.

\section{INTRODUÇÃO}

A espécie Nicotiana glauca Graham (fumobravo; charuto-do-rei) é uma Solanaceae arbustiva, com altura variando de 2-6 m (VIGNOLI-SILVA e MENTZ, 2005; MORAES et al., 2009). Nativa da Argentina e Bolívia (GOODSPEED et al., 1954; NEE, 1986), atualmente é encontrada crescendo espontaneamente em zonas áridas e semiáridas de diferentes partes do globo (SANZ-ELORZA et al., 2010), a exemplo da Arábia Saudita (ALSHAHRANI, 2008), Chile (UGARTE et al., 2011), Chipre (CHRTEK e SLAVIK, 1993), Estados Unidos (BURGESS et al., 1991; SCHUELLER, 2004) e México (DÍAZ e LEÓN, 2008). Em alguns países como a Austrália (FLORENTINE e WESTBROOKE, 2005; FLORENTINE et al., 2006), Croácia (BOGDANOVIC et al., 2006), Espanha (SANZ-ELORZA et al., 2004), Namíbia (SHAPAKA et al., 2008) e Portugal (PORTUGAL, 1999), a espécie é reconhecida como exótica invasora.

Todas as partes da planta são tóxicas para os seres humanos (SCHENKEL et al., 2003) e animais (CUNNINGHAM et al., 1981; BOTHA et al., 2011) em razão da presença do alcaloide anabasina (RAGONESE, 1955). Nicotiana glauca altera a disponibilidade de recursos hídricos, devido a suas altas taxas de transpiração, podendo comprometer assim, o desenvolvimento da comunidade nativa em áreas de clima seco (BRANDES, 2000; SANZELORZA et al., 2004).

No Brasil, a espécie já foi coletada em algumas regiões (CRIA, 2012b; STEHMANN et al., 2012) e citada em alguns trabalhos científicos sempre com baixa representatividade - poucos indivíduos (PEGADO et al., 2006; MORAES et al., 2009). Contudo, nas Áreas Diretamente Afetadas (ADA) das obras do Projeto de Integração do Rio São Francisco com Bacias Hidrográficas do Nordeste Setentrional (PISF) (MIN, 2004), particularmente no Eixo Leste do mesmo, Nicotiana glauca apresenta comportamento agressivo, diferindo completamente do que era apontado para ela até o momento. Por toda a extensão do referido ambiente, que tem por volta de $220 \mathrm{~km}$ de comprimento (MIN, 2004), e 10-20 m de largura de ambos os lados do canal, a espécie pode ser observada formando populações monodominantes extremamente adensadas (FABRICANTE e SIQUEIRA-FILHO, 2012), o que deve potencializar os problemas causados pela espécie sobre a biota, além de aumentar as chances de casos de intoxicação humana e animal.

Assim, o presente estudo buscou avaliar atributos ecológicos de Nicotiana glauca, possibilitando com os seus resultados a tomada de decisões adequadas para o controle ou manejo da espécie. Para tanto, foram investigados aspectos ligados à estrutura (densidade, biometria e diversidade) e reprodução (produção de frutos, propágulos e germinabilidade) da espécie, avaliado o potencial aleloquímico da planta e estimada a área de ocorrência potencial do táxon no Brasil.

\section{MATERIAL E MÉTODOS}

\section{Área de estudo}

A área de estudo ( $8^{\circ} 7^{\prime} 38,5^{\prime} \mathrm{S}$ e $37^{\circ} 26^{\prime \prime} 54,3$ " $\mathrm{W}, 512 \mathrm{~m}$ de altitude média), localizase no município de Custódia - PE, no Eixo Leste do PISF (MIN, 2004). As informações coletadas por meio de entrevista estruturada realizada com moradores da região, e funcionários da empresa executora da obra no trecho do estudo, não permitiram estabelecer o local por onde se iniciou o processo de bioinvasão de Nicotiana glauca. Foi consenso que a espécie já era observada em ambientes ruderais e perturbados nos arredores da área de estudo, porém, com baixa frequência. Ainda, que a espécie mudou seu "comportamento" de forma abrupta com o advento das obras do PISF, passando a ocorrer com altas densidades em todo o seu Eixo Leste.

A vegetação do local, composta pela Caatinga - Savana Estépica (IBGE, 1992), foi suprimida há um pouco mais de quatro anos para a construção do canal do referido projeto. O clima regional é o semiárido quente, BSh, segundo a classificação de Köppen-Geiger, com precipitação anual de 431,8 mm (CPRM, 2005).

Os solos predominantes na área são os Luvissolos Crômicos Órticos, mas existem outras classes ao longo do canal. Por toda a sua extensão, os solos encontram-se bastante compactados em razão do trânsito de maquinários pesados, e tiveram 
removidos ou soterrados seus horizontes "O" e "A" por material oriundo da escavação dos canais (bota-fora - solos, cascalho e rochas de diferentes tamanhos).

\section{Estrutura da população e diversidade da comunidade}

A estrutura populacional de Nicotiana glauca foi avaliada em 10 parcelas (MULLERDOMBOIS e ELLEMBERG, 1974), com dimensões de $25 \mathrm{~m}^{2}$, que foram alocadas aleatoriamente pelo sítio estudado. Nestas unidades amostrais, todos os indivíduos foram amostrados e os da espécie Nicotiana glauca ainda tiveram aferidos seu DNS (diâmetro ao nível do solo) e altura (RODAL et al., 1992) e, posteriormente, foram classificados quanto ao seu estádio ontogenético em adultos e regenerantes. Aqueles que apresentavam material reprodutivo foram considerados adultos e os demais foram considerados regenerantes.

A densidade (DA) foi calculada para cada estádio ontogenético, assim como para toda a população (KENT e COKER, 1999). Os parâmetros biométricos dos indivíduos de Nicotiana glauca foram distribuídos em classes de frequência, cuja amplitude foi de $1 \mathrm{~cm}$ para o DNS e de $1 \mathrm{~m}$ para a altura.

A diversidade e a equitatividade da área foram estimadas por meio dos índices de ShannonWeaver $\left(H^{\prime}\right)$ (SHANNON e WEAVER, 1949) e de Pielou (J) (PIELOU, 1977), respectivamente.

\section{Reprodução}

Foram selecionados de forma aleatória 10 indivíduos reprodutivos de Nicotiana glauca para a contagem do número de frutos por planta. Também foram coletados 100 frutos de diferentes matrizes para o conhecimento do número de sementes por fruto.

Visando aferir o potencial germinativo dos propágulos de Nicotiana glauca, quatro repetições de 100 sementes, foram acondicionadas sobre papel gerbox em caixa de polietileno transparente e colocadas em câmera de germinação do tipo B.O.D., a uma temperatura constante de $25 \pm 1^{\circ} \mathrm{C}$, cujo substrato foi mantido na capacidade de campo até o final do experimento (BRASIL, 2009). A germinação foi verificada diariamente até a sua estabilização, sendo consideradas germinadas as sementes que apresentavam $2 \mathrm{~mm}$ de protrusão radicular (BRASIL, 2009).

\section{Alelopatia}

Folhas localizadas em diferentes alturas nas copas de indivíduos de Nicotiana glauca foram coletadas e secas a uma temperatura de $50^{\circ} \mathrm{C}$ em estufa de circulação forçada. Para a preparação dos estratos, a folhas secas foram trituradas e diluídas em água destilada. As concentrações utilizadas foram: 0 (testemunha), 5, 10, 15, e 20\% (p/v). As soluções foram colocadas em recipientes de vidro envolvidos por papel-alumínio para evitar fotodegradação, permanecendo em repouso por 24 horas. Após esse período, as soluções foram filtradas e utilizadas nos bioensaios.

Para cada concentração dos extratos, utilizaram-se os mesmos procedimentos descritos para o teste de germinação. A espécie utilizada para os testes foi Lactuca sativa L. (alface), variedade "Grand Rapids TBR", com porcentagem de germinação de $99 \%$.

Avaliou-se o percentual de germinação e o tamanho total e a matéria seca das plântulas (LABOURIAU e VALADARES, 1976; MAGUIRE, 1962). Os dados foram submetidos à análise de variância e as médias comparadas pelo teste Tukey $(\mathrm{p} \leq 0,05)($ TUKEY, 1959).

\section{Modelagem de nicho ecológico}

A área potencial de invasão de Nicotiana glauca no Brasil (susceptibilidade de ocorrência) foi avaliada por meio da análise de modelagem de nicho ecológico, baseada em variáveis climáticas (precipitação e temperatura) (CRIA, 2012a). Os pontos de georreferenciamento de ocorrência da espécie foram obtidos na base de dados do SpeciesLink (CRIA, 2012b).

$\mathrm{O}$ algoritmo utilizado para a modelagem foi a Máxima Entropia (PHILLIPS et al., 2006). Para avaliar o comportamento dos dados climáticos dos locais de ocorrência de Nicotiana glauca, foram construídos diagramas Boxplot (CLEVELAND, 1994). As informações foram obtidas na planilha gerada pelo openModeller $1.1^{\circ}$ (CRIA, 2012a).

\section{Programas estatísticos}

As análises estatísticas foram executadas utilizando-se os softwares Past $1.12^{\circ}$ (HAMMER et al., 2003), SSC-Stat $2.18^{\circ}$ (STERN et al., 2007), 
Maxent 3.3.3k ${ }^{\odot}$ (MAXENT, 2012) e openModeller $1.1^{\odot}$ (CRIA, 2011).

\section{RESULTADOS E DISCUSSÃO}

\section{Estrutura da população}

O número médio de indivíduos nas parcelas foi de 93,2 \pm 59 , dos quais $61,6 \pm 54,7$ pertencentes à categoria regenerantes e 31,6 $\pm 17,8$ a categoria adultos. A densidade total da espécie foi estimada em $37.280 \pm 2.442,3$ indivíduos ha, sendo 24.640 $\pm 1.985,5$ regenerantes ha e $12.640 \pm 1.422,1$ adultos ha.

A abundância de espécimes observada neste estudo foi superior às apresentadas por Florentine e Westbrooke (2005) e Florentine et al. (2006) em área invadida por Nicotiana glauca na Austrália, assim como as encontradas para a espécie na Croácia (BOGDANOVIC et al., 2006). Na sua área de distribuição natural, Nicotiana glauca ocorre em manchas isoladas com 15-70 indivíduos (NATTERO et al., 2011), diferindo do que vem acontecendo nas áreas do PISF, em que o táxon forma extensas populações contíguas e com grande número de indivíduos.

Para Ziller (2001), determinados ambientes possuem características que os tornam mais suscetíveis à invasão biológica do que outros. Alguns atributos apontados pela autora estão representados nas áreas do PISF, tais como: sítios intensamente degradados, baixa biodiversidade e ausência ou baixa quantidade de inimigos da espécie.

A densidade de Nicotiana glauca também foi mais elevada do que as das espécies invasoras de maior importância na Caatinga até o momento. Em áreas de mata ciliar nos Estados do Rio Grande do Norte e Paraíba, Prosopis juliflora DC. (algaroba) obteve uma variação de 1.954,5-3.450 indivíduos ha (ANDRADE et al., 2008; ANDRADE et al., 2009). Já nas margens do rio Paraíba, município de Natuba - PB, Sesbania virgata (Cav.) Pers. (sesbânia) exibiu uma densidade de 11.560 indivíduos ha (SOUZA et al., 2011).

O DNS e altura média da população foram de $1,08 \pm 1,01 \mathrm{~cm}$ e de $0,73 \pm 0,89 \mathrm{~m}$, respectivamente, sendo de $0,69 \pm 0,21 \mathrm{~cm}$ e de $0,31 \pm 0,15 \mathrm{~m}$ para os regenerantes, e de $2,12 \pm 0,49 \mathrm{~cm}$ e de $1,75 \pm$ $0,6 \mathrm{~m}$ para os adultos. A espécie apresentou maior porcentagem de indivíduos nas primeiras classes de frequência diamétrica e hipsométrica, com redução constante nesse número, até atingirem os menores valores nas menores classes, formando assim curvas exponenciais em " $\mathrm{J}$ " invertido, com altos valores de coeficiente de determinação $\left(\mathrm{R}^{2}\right)$ (Figura 1), o que caracteriza uma população estável, autorregenerante (SCOLFORO et al., 1998) e assim, sugere estar completamente adaptada às condições da região.

Quatro espécies, pertencentes a três famílias, foram amostradas em meio aos indivíduos de Nicotiana glauca (Tabela 1). A diversidade de Shannon-Weaver $\left(H^{\prime}\right)$ da área estudada foi de 0,3007 e a equitatividade de Pielou (J) foi de 0,1869 .

Estes valores foram semelhantes aos encontrados para áreas de caatinga invadidas por P. juliflora (ANDRADE et al., 2008; 2009; 2010) e Calotropis procera (Aiton) W.T.Aiton (FABRICANTE et al., 2013). Ao compararem seus
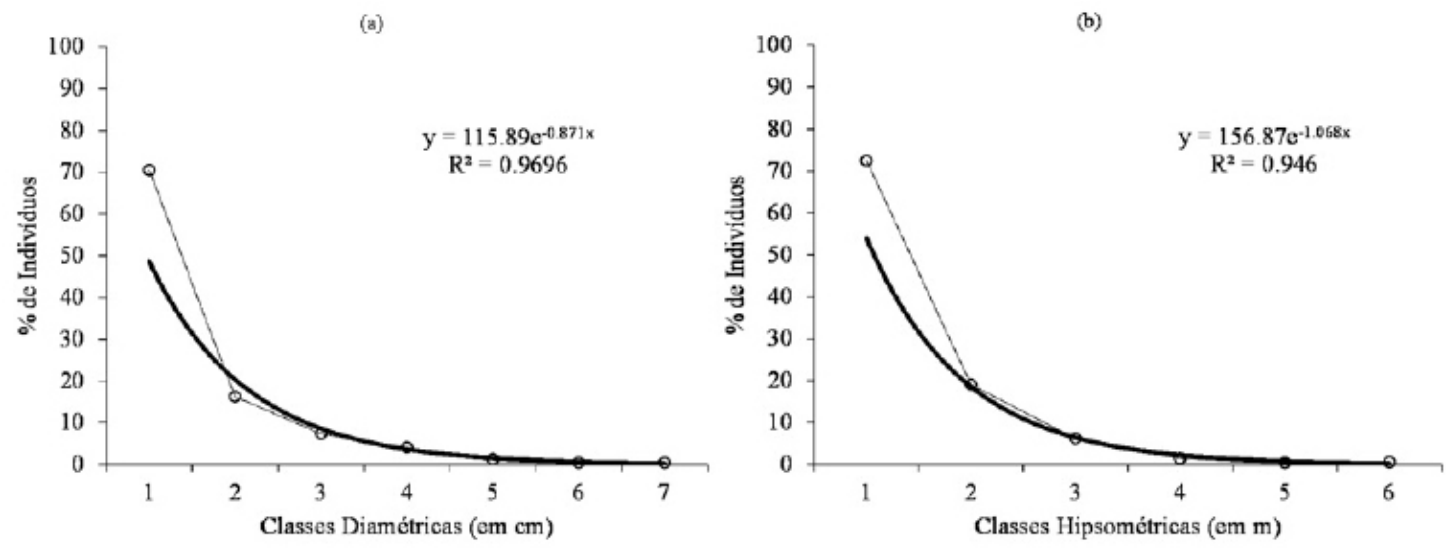

FIGURA 1: Distribuição da abundância de indivíduos (em \% de contribuição) de Nicotiana glauca Graham em classes de frequência diamétrica (a) e hipsométrica (b).

FIGURE 1: Distribution of numbers of individuals (\%) of Nicotiana glauca Graham in diameter classes (a) and height classes (b). 
TABELA 1: Lista de famílias e espécies amostradas em uma área invadida por Nicotiana glauca Graham em Custódia - PE. Sendo $\mathrm{n}$ = número de indivíduos; $\mathrm{N}$ = número de parcelas com a presença da espécie.

TABLE 1: List of families and species sampled in an area invaded by Nicotiana glauca Graham in Custody, PE. Where $\mathrm{n}=$ number of individuals, $\mathrm{N}=$ number of plots with the presence of the species.

\begin{tabular}{cccc}
\hline Família & Espécie & $\mathrm{n}$ & $\mathrm{N}$ \\
\hline Cleomaceae & Tarenaya spinosa (Jacq.) Raf. & 2 & 2 \\
Malvaceae & Sida galheirensis Ulbr. & 78 & 9 \\
& Melochia pyramidata L. & 1 & 1 \\
Papaveraceae & Argemone mexicana L. & 1 & 1 \\
Solanaceae & Nicotiana glauca Graham & 932 & 10 \\
\hline Total & & 1014 & 10 \\
\hline
\end{tabular}

resultados com os de trabalhos desenvolvidos em comunidades autóctones, os autores observaram sensíveis diferenças entre os sítios, sendo a diversidade sempre menor naqueles invadidos, devendo-se a isso, a baixa riqueza de espécies, associada à baixa equitatividade na distribuição da abundância de indivíduos entre as entidades taxonômicas. $\mathrm{Na}$ área do presente estudo, além de apresentar apenas cinco espécies, um pouco mais de $90 \%$ da comunidade estavam representadas por espécimes de Nicotiana glauca.

\section{Reprodução}

Foram contabilizados $2.120,9 \pm 939,6$ frutos por planta e $643,9 \pm 49,9$ sementes por fruto. A porcentagem de germinação foi de $95 \%$. Valendo-se desses valores, estima-se que uma planta possa produzir um pouco mais de 1.300 .000 sementes. Brandes (2000) apontou uma variação de 10.000-1.000.000 de propágulos por indivíduo de Nicotiana glauca.

Nas áreas de dispersão natural, Nicotiana glauca floresce de setembro a abril (NATTERO et al., 2011), já em áreas invadidas, a floração e frutificação ocorrem ao longo de todo o ano (SANZ-ELORZA et al., 2004; VIGNOLI-SILVA e MENTZ, 2005), o que também pôde ser observado nas áreas do PISF (SIQUEIRA-FILHO, J.A., observação pessoal). Os frutos são caracterizados por cápsulas com fendas verticais, através das quais as sementes são dispersas pelo vento e ou pela água (BRANDES, 2000; SANZ-ELORZA et al., 2004; BOGDANOVIC et al., 2006).

Brandes (2000), reporta que nas últimas décadas a construção de estradas tem papel relevante

TABELA 2: Porcentagem de germinação, comprimento (em centímetro) e peso da matéria seca (em gramas) de plântulas de Lactuca sativa L. em razão das concentrações dos estratos aquosos de Nicotiana glauca Graham.

TABLE 2: Germination percentage, length (in centimeters) and dry matter (in grams) of seedlings of Lactuca sativa L. because the concentrations of aqueous strata of Nicotiana glauca Graham.

\begin{tabular}{cccc}
\hline Tratamento & \multicolumn{3}{c}{ Variáveis } \\
\cline { 2 - 4 } & $\%$ de Germinação & Comprimento & Matéria Seca \\
\hline $0 \%$ & $98,8 \pm 0,5 \mathrm{a}$ & $5 \pm 0,08 \mathrm{a}$ & $0,1284 \pm 0,01 \mathrm{a}$ \\
$5 \%$ & $78,5 \pm 11,26 \mathrm{~b}$ & $2,5 \pm 0,45 \mathrm{~b}$ & $0,0826 \pm 0,004 \mathrm{~b}$ \\
$10 \%$ & $12,5 \pm 9,88 \mathrm{c}$ & $0,6 \pm 0,29 \mathrm{c}$ & $0,0780 \pm 0,002 \mathrm{~b}$ \\
$15 \%$ & $0 \mathrm{~d}$ & $0 \mathrm{~d}$ & $0 \mathrm{c}$ \\
$20 \%$ & $0 \mathrm{~d}$ & $0 \mathrm{~d}$ & $0 \mathrm{c}$ \\
\hline
\end{tabular}

Em que: Médias seguidas de mesma letra não diferem entre si a uma probabilidade maior ou igual a 5\% segundo o teste Tukey. 
na dispersão da espécie nas Ilhas Canárias. Este fato também foi citado pelos entrevistados das empresas responsáveis pelas obras do PISF.

Assim, a grande produção de sementes diminutas com altas taxas de germinação, em conjunto com a dispersão realizada pelos maquinários utilizados na obra, parecem ser a razão para o rápido estabelecimento de Nicotiana glauca por toda a extensão do Eixo Leste do PISF.

\section{Alelopatia}

Todas as variáveis das plântulas $(\%$ de germinação, comprimento e peso da matéria seca) de Lactuca sativa apresentaram diferenças significativas a partir da primeira concentração do extrato e foram nulas a partir de $15 \%$ de concentração (Tabela 2), sugerindo a presença de aleloquímicos nas folhas de Nicotiana glauca.

Além de afetar de maneira incisiva a germinação e desenvolvimento de plântulas de $L$. sativa como demonstrado nesse estudo, Nicotiana glauca também é capaz de causar danos a plantas em estágios de desenvolvimento mais tardios, podendo até mesmo ocasionar sua morte, conforme ficou evidenciado nos resultados apresentados por Alshahrani (2008).

A produção de substâncias alelopáticas parece assumir um papel importante na manutenção de populações monodominantes de espécies invasoras. A presença de aleloquímicos tem sido verificada em grande parte das espécies invasoras já estudadas (PERDOMO e MAGALHÃES, 2007; ROSA et al., 2007; RABÊLO et al., 2008; CHAPLA e CAMPOS, 2010).

\section{Modelagem de nicho ecológico}

A espécie apresentou alta probabilidade de ocorrência em todo o Domínio da Savana Estépica (IBGE, 1992), probabilidade variando de baixa a alta em regiões do país dominadas pelo Cerrado Savana (IBGE, 1992) e pelas Florestas Estacionais Semideciduais e Deciduais (IBGE, 1992). A análise também apontou probabilidade de ocorrência variável no litoral nordestino, do Espirito Santo e do Rio de Janeiro. No extremo Sul do País, e em porções da região amazônica onde ocorrem as Florestas Ombrófilas Abertas (IBGE, 1992), a espécie apresentou de baixa a média probabilidade
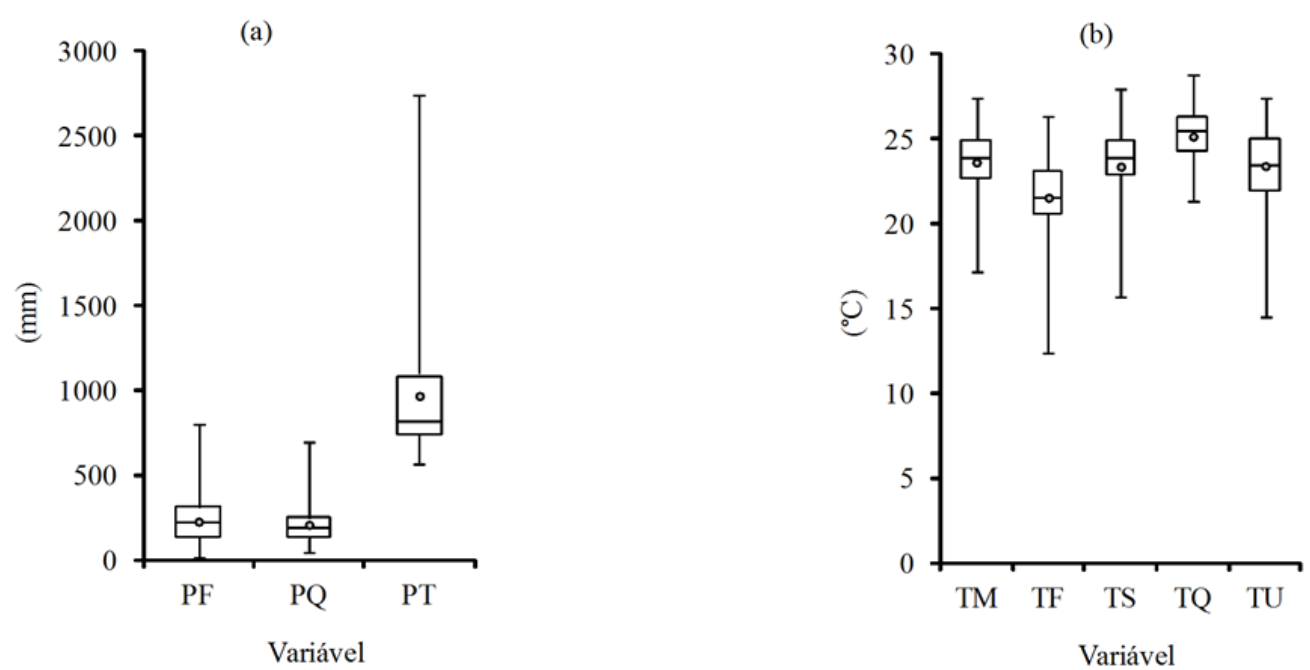

FIGURA 2: Boxplot da precipitação (a) e da temperatura (b) dos locais de ocorrência de Nicotiana glauca Graham. Sendo: $\mathrm{PF}=$ precipitação no período mais frio; $\mathrm{PQ}=$ precipitação no período mais quente; $\mathrm{PT}=$ precipitação total; $\mathrm{TM}=$ temperatura média; $\mathrm{TF}=$ temperatura no período mais frio; $\mathrm{TS}=$ temperatura no período mais seco; $\mathrm{TQ}=$ temperatura no período mais quente; $\mathrm{TU}=$ temperatura no período mais úmido.

FIGURE 2: Boxplot of precipitation (a) and temperature (b) of the places of occurrence of Nicotiana glauca Graham. Where: $\mathrm{PF}=$ precipitation during the cold period, $\mathrm{PQ}=$ precipitation during warmer period; $\mathrm{PT}=$ total precipitation $\mathrm{TM}=$ average temperature, $\mathrm{TF}=$ temperature in the coldest period, $\mathrm{TS}=$ temperature in the driest period; $\mathrm{TQ}=$ temperature during hottest period; $\mathrm{TU}=$ temperature during the humid period. 
de ocorrência.

As amplitudes de tolerância da espécie à precipitação e temperatura apontam que Nicotiana glauca é euri-hídrica e euritérmica. A precipitação no período mais frio variou de 10 a $793 \mathrm{~mm}$, no período mais quente de 44 a $690 \mathrm{~mm}$ e a total variou de 568 a $2727 \mathrm{~mm}$. Quanto à temperatura, a média variou de 17,2 a $27,3^{\circ} \mathrm{C}$, sendo que no período mais frio variou de 12,3 a $26,2^{\circ} \mathrm{C}$, no período mais seco de 15,6 a $27,9^{\circ} \mathrm{C}$, no período mais quente de 21,3 a $28,8^{\circ} \mathrm{C}$ e, no período mais úmido de 14,5 a $27,3^{\circ} \mathrm{C}$ (Figura 2).

Estudo anatômico e morfológico realizado com a espécie na África do Sul indicou a presença de estruturas semelhantes às encontradas em plantas de regiões áridas (MHINANA et al., 2010). Florentine e Westbrooke (2005) observaram que as características ecológicas apresentadas pela espécie em área invadida na Austrália são análogas às de outras plantas daninhas de zonas secas. $\mathrm{Na}$ África do Sul (VAHRMEIJER, 1981), Croácia (BOGDANOVIC et al., 2006) e Espanha (SANZELORZA et al., 2004), Países onde a espécie é invasora, Nicotiana glauca ocorre em ambientes semelhantes aos apontados neste estudo.

Em vista destes fatos, é possível apontar o "Domínio das Florestas Sazonais Tropicais" (PRADO, 2003), como sendo a zona mais susceptível à invasão biológica por Nicotiana glauca no Brasil.

\section{CONCLUSÕES}

As características biofísicas vigentes nas áreas do PISF (degradação da paisagem, baixa diversidade biológica, ausência de inimigos da espécie e facilitadores para dispersão), somadas aos atributos intrínsecos da espécie (populações com altas densidades, maturação precoce dos indivíduos estabelecidos, alta produção de propágulos e produção de substâncias alelopáticas), concorrem para explicar a explosão censitária observada para Nicotiana glauca na região;

A avaliação de áreas susceptíveis à ocorrência da espécie indicou as regiões semiáridas e subúmidas do país como as mais propícias ao estabelecimento de processos de bioinvasão por Nicotiana glauca;

Os resultados deste estudo demonstram a necessidade do controle imediato da espécie nas áreas do PISF e, subsidiam a formulação de meios para evitar novos casos de invasão biológica por Nicotiana glauca em outras partes do Brasil.

\section{AGRADECIMENTOS}

Os autores agradecem ao Ministério da Integração Nacional pela logística.

\section{REFERÊNCIAS BIBLIOGRÁFICAS}

ALSHAHRANI, S.T. Effect of aqueous extract of the invasive species Tobacco (Nicotiana glauca L.) on seedlings growth of Juniper (Juniperus procera L.). Emirates Journal of Food and Agriculture, v. 20 , n. 2 , p. $10-17$, fev. 2008.

ANDRADE, L.A.; FABRICANTE, J.R.; ALVES, A.S. Algaroba (Prosopis juliflora (Sw.) DC.): Impactos sobre a Fitodiversidade e Estratégias de Colonização em Área Invadida na Paraíba, Brasil. Natureza e Conservação, v. 6, n. 1, p. 46-54, jan. 2008.

ANDRADE, L.A.; FABRICANTE, J.R.; OLIVEIRA, F.X. Invasão biológica por Prosopis juliflora (Sw.) DC.: impactos sobre a diversidade e a estrutura do componente arbustivo-arbóreo da caatinga no Estado do Rio Grande do Norte, Brasil. Acta Botanica Brasilica, vol. 23, n. 4, p. 935-943, out. 2009.

ANDRADE, L.A.; FABRICANTE, J.R.; OLIVEIRA, F.X. Impactos da Invasão de Prosopis juliflora (Sw.) DC. (Fabaceae) sobre o Estrato Arbustivo-Arbóreo em Áreas de Caatinga no Estado da Paraíba, Brasil. Acta Scientiarum. Biological Sciencies, vol. 32, n. 3: 249-255, jul. 2010. BOGDANOVIC, S. et al. Nicotiana glauca Graham (Solanaceae), a new invasive plant in Croatia. Acta Botanica Croatica, vol. 65, n. 2, p. 203-209, abr. 2006.

BOTHA C.J. et al. Nicotiana glauca poisoning in ostriches (Struthio camelus). Journal of the South African Veterinary Association, vol. 82, n. 2, p. 116-119, fev. 2011.

BRANDES, D. Nicotiana glauca als invasive Pflanze auf Fuerteventura. Dietmar Brandes (Hrsg.): Adventivpflamen. Beiträge zu Biologie, Vorkommen und Ausbreitungsdynamik von Archäophyten und Neophyten in Mitteleuropa. Tagungsbehcht des Braunschweiger Kolloquiums vom 3. Braunschweig. Braunschweiger Geobotanische Arbeiten, v. 8, p. 39-57, jan. 2000. BRASIL. Regras para análise de sementes. Ministério da Agricultura, Pecuária e Abastecimento. Brasília, Brasil. 2009. 399 p.

BURGESS, T.L.; BOWERS, J.E.; TURNER, R.M. Exotic plants at the desert laboratory, Tucson, 
Arizona. Madroño, vol. 38, n. 2, p. 96-114, 1991. CHAPLA, T.E.; CAMPOS, J.B. Allelopathic Evidence in Exotic Guava (Psidium guajava L.). Brazilian archives of Biology and Technology, v. 53, n. 6. p. 1359-1362, jun. 2010.

CHRTEK, J.; SLAVIK, B. Contribution to the flora of Cyprus. Flora Mediterranea, v. 3, p. 239-259, 1993.

CLEVELAND, W.S. The elements of graphing data. Hobart Press, 2ed. 1994. 297 p.

CPRM. Projeto cadastro de fontes de abastecimento por água subterrânea Diagnóstico do município de Custódia, estado de Pernambuco. Recife: CPRM/PRODEEM, 2005. $12 \mathrm{p}$.

CRIAa. Openmodeller. Disponível em: http:// openmodeller.cria.org.br/.Acesso em 10.maio.2012. CRIAb. SpeciesLink. Disponível em: http://splink. cria.org.br/. Acesso em 02.fevereiro.2012.

CUNNINGHAM, G.M. et al. Plants of Western New South Wales. N.S.W. Government Printing Office, Sydney. 1981. p.776.

DÍAZ, G.M.; LEON, J.J. Composición florística em los huertos de Nogal pacanero em México. Instituto Nacional de Investigaciones Forestales, Agrícolas y Pecuárias. 1 ed. p. 5-180. 2008.

FABRICANTE, J.R.; SIQUEIRA-FILHO, J.A. Exotic and Invasive Plants of the Caatingas of the São Francisco River. In: SIQUEIRA-FILHO, J.A.. (Org.). Flora of the Caatingas of the São Francisco River: Natural History and Conservation. 1ed. Rio de Janeiro, RJ: Andrea Jakobsson Estúdio Editorial. v. 1, p. 366-393. 2012.

FABRICANTE， J.R.; OLIVEIRA， M.N.A.; SIQUEIRA-FILHO, J.A. Aspectos da ecologia de Calotropis procera (Aiton) W.T.Aiton (Apocynaceae) em uma área de caatinga alterada pelas obras do Projeto de Integração do Rio São Francisco em Mauriti, CE. Rodriguesia. vol. 64, p. 647-654, jul. 2013.

FLORENTINE, S. K. et al. The arid land invasive weed Nicotiana glauca R. Graham (Solanaceae): Population and soil seed bank dynamics, seed germination patterns and seedling response to flood and drought. Journal of Arid Environments, v. 66, p. 218-230, jun. 2006.

FLORENTINE, S.K.; WESTBROOKE, M.E. Invasion of the noxious weed Nicotiana glauca $R$. Graham after an episodic flooding event in the arid zone of Australia. Journal of Arid Environments, v. 60 , p. $531-545$, jan. 2005.

GOODSPEED, T.H. The genus Nicotiana. Chronica
Botanica. Waltham, Massachusetts, USA. 1954. HAMMER, O.; HAPER, D.A.T.; RYAN, P.D. PAST: Paleontological Statistics Software package for education and data analysis. Palaeontologia Electronica. Oslo. 2003.

IBGE. Manual técnico da vegetação brasileira. Rio de Janeiro. Série Manuais Técnicos em Geociências 1. 1992. 92 p.

KENT, M.; COKER, P. Vegetation Description and Analysis - a pratical approach. Chichester: John Wiley e Sons. 1999. 363 p.

LABORIAU, L.G.; VALADARES, M.B. On the germination of seeds of Calotropis procera. Anais da Academia Brasileira de Ciências, v. 48, p. 174-186, out. 1976.

MAGUIRE, J.D. Speed of germination-aid in selection and evaluation for seedling emergence and vigor. Crop science, vol. 2, n. 1, p. 176-177, jan. 1962.

MAXENT. Software for species habitat modeling. Version: 3.3.3k. Disponível em: http://www. cs.princeton.edu/ schapire/maxent/. Acesso em 10.maio.2012.

MHINANA, Z.; MAYEKISO, B.; MAGWA, M.L. Anatomy and morphology of Nicotiana glauca with regard to its crystals characterization. African Journal of Plant Science, v. 4, n. 6, p. 172-178, jun. 2010.

MIN. Projeto de Integração do Rio São Francisco com Bacias Hidrográficas do Nordeste Setentrional. Relatório de Impacto Ambiental (RIMA). Brasilia: Ministério da integração Nacional, 2004. 136 p.

MORAES, A.O et al. Família Solanaceae nos "Inselbergues" do semi-árido da Bahia, Brasil. Iheringia, Série Botânica, v. 64, n. 2, p. 109-122, abr. 2009.

MULLER-DOMBOIS, D.; ELLEMBERG, H. Aims and methods of vegetation ecology. John Wiley e Sons. New York. 1974. 547 p.

NATTERO, J.; SÉRSIC, A.N.; COCUCCI, A. A. Geographic variation of floral traits in Nicotiana glauca: Relationships with biotic and abiotic factors. Acta Oecologica, v. 37, n. 5, p. 503-511, mai. 2011. NEE, M. Flora de Veracruz: Solanaceae. México: Instituto National de Investigaciones. Veracruz. pt. 1, 1986. $191 \mathrm{p}$.

PEGADO, C.M.A. et al. Efeitos da invasão biológica de algaroba - Prosopis julifl ora (Sw.) DC. sobre a composição e a estrutura do estrato arbustivoarbóreo da caatinga no Município de Monteiro, PB, Brasil. Acta Botanica Brasilica, v. 20, n. 4, 
p. 887-898, out. 2006 .

PERDOMO, M.; MAGALHÃES, L.M.S. Ação alelopática da jaqueira (Artocarpus heterophyllus) em laboratório. Floresta e Ambiente, v. 14, n. 1, p. 52-55, jan. 2007.

PHILLIPS, S.J.; ANDERSON, R.P.; SCHAPIRE, R.E. Maximum entropy modeling of species geographic distributions. Ecological Modeling, v. 190, p. 231-259, 2006.

PIELOU, U.C. Mathematical diversity. John Wiley e Sons. New York. 1977. 385 p.

PORTUGAL. Decreto-Lei n. 565/99 de 21 de Dezembro de 1999. Diário da República - I Série-A n. 295. Ministério do Ambiente. p. 9100-9114. 1999. PRADO, D.E. As caatingas do Brasil. Leal, I.R., Tabarelli, M., Silva, J.M.C. (eds.). Ecologia e conservação da Caatinga. Ed. Universidade Federal de Pernambuco, Recife. p. 3-73, 2003.

RABÊLO, G.O. et al. Potencial alelopático de Bidens pilosa L. na germinação e no desenvolvimento de espécies cultivadas. Revista Científica de Faminas, v. 4, n. 1, p. 1-8, jan. 2008.

RAGONESE, A.E. Plantas toxicas para el ganado en la region central Argentina. Revista de la Faculd de Agronomia, v. 31, p. 133-336. 1955.

RODAL, M.J.N.F.; SAMPAIO, E.V.S.B.; FIGUEIREDO, M.A. Manual sobre métodos de estudos florísticos e fitossociológicos-ecossistema caatinga. Brasília: Sociedade Botânica do Brasil 72. 1992.

ROSA, D.M. et al. Efeito dos Extratos de Tabaco, Leucena e Sabugueiro sobre a Germinação de Panicum maximum Jaqc. Revista Brasileira de Biociências, v. 5, n. 2, p. 444-446, 2007.

SANZ-ELORZA, M., DANA, E.D.; SOBRINO, E. Atlas de lãs plantas alóctonas invasoras de España. Dirección General para la Biodiversidad, Madrid, Spain. 2004.

SANZ-ELORZA, M. et al. Invasiveness of alien vascular plants in six arid zones of Europe, Africa and America. Lazaroa, v. 31, p. 109-126, 2010.

SCHENKEL, E.P. et al. Plantas Tóxicas. In: SIMÕES, C.M.O.; SCHENKEL, E.P.; GOSMANN, G.; MELLO, J.C.P.; MENTZ, L.A.; PETROVICK, P.R. (org.). Farmacognosia: da planta ao medicamento. 5. Ed. Rev. Ampl. Porto Alegre:
Editora da universidade/UFRGS; Florianópolis. p. 959-993. 2003.

SCHUELLER, S.K. Self-Pollination in Island and Mainland populations of the introduced hummingbird-pollinated plant, Nicotiana glauca (Solanaceae). American Journal of Botany, v. 91, n. 5, p. 672-681, mai. 2004.

SCOLFORO, J.R.S.; PULZ, F.A.; MELLO, J.M. Modelagem da produção, idade das florestas nativas, distribuição espacial das espécies e a análise estrutural. In: Scolforo, J.R.S. (Org.) Manejo Florestal: UFLA/ FAEPE. p. 189-245. 1998.

SHANNON, C.E.; WEAVER, W. The Mathematical Theory of Communication. Urbana: University Illinois Press. 1949.

SHAPAKA, T.N.; CUNNINGHAM, P.L.; JOUBERT, D.F. Invasive alien plants in the Daan Viljoen Game Park. Windhoek, Namibia. Dinteria, v. 30, p. 19-32, jan. 2008.

SOUZA, V.C. et al. Avaliação populacional de Sesbania virgata (Cav.) Pers. (Fabaceae Lindl.), nas margens do rio Paraíba Revista Brasileira de Ciências Agrárias, v. 6, n. 2, p. 314-320, abr. 2011. STEHMANN, J.R. et al. Solanaceae. In: Lista de Espécies da Flora do Brasil. Jardim Botânico do Rio de Janeiro. Disponível em: http://floradobrasil. jbrj.gov.br/2012/FB021155. Acesso em: 10.mai.2012.

STERN, R.; LEIDI, S.; GRAYER, C. SSC-Stat 2.18 tutorial. Statistical Services Centre. The University of Reading, UK. 2007. 28 p.

TUKEY, J.W. A Quick, Compact, Two-Sample Test to Duckworth.s Specications. Technometrics, v. 1, n. 31, jan. 1959.

UGARTE, E. et al. Vascular alien flora, Chile. Check List, v. 7, n. 3, p. 365-382, 2011.

VAHRMEIJER, J. Poisonous plants of southern Africa that cause stock losses. Tafelberg Publishers. Cape Town. 1981.

VIGNOLI-SILVA, M.; MENTZ, L.A. O gênero Nicotiana L. (Solanaceae) no Rio Grande do Sul, Brasil. Iheringia, Série Botânica, v. 60 , n. 2, p. 151-173, abr. 2005.

ZILLER, S.R. Plantas exóticas invasoras: a ameaça da contaminação biológica. Ciência Hoje, v. 30, n. 178, p. 77-79, 2001. 